\title{
Demonstrated Wavelength Portability of Raman Reference Data for Explosives and Chemical Detection
}

\author{
Timothy J. Johnson, ${ }^{1}$ Yin-Fong Su, ${ }^{1}$ Kristin H. Jarman, ${ }^{1}$ Brenda M. Kunkel, ${ }^{1}$ \\ Jerome C. Birnbaum, ${ }^{1}$ Alan G. Joly, ${ }^{1}$ Eric G. Stephan, ${ }^{1}$ Russell G. Tonkyn, ${ }^{1}$ \\ Robert G. Ewing, ${ }^{1}$ and Glen C. Dunham ${ }^{2}$ \\ ${ }^{1}$ Pacific Northwest National Laboratory, P.O. Box 999, Richland, WA 99354, USA \\ ${ }^{2}$ Dunham Research, 3251 Kemptown Church Road, Monrovia, MD 21770, USA \\ Correspondence should be addressed to Timothy J. Johnson, timothy.johnson@pnnl.gov
}

Received 18 February 2012; Revised 13 April 2012; Accepted 17 April 2012

Academic Editor: Augustus Way Fountain

Copyright () 2012 Timothy J. Johnson et al. This is an open access article distributed under the Creative Commons Attribution License, which permits unrestricted use, distribution, and reproduction in any medium, provided the original work is properly cited.

\begin{abstract}
As Raman spectroscopy continues to evolve, questions arise as to the portability of Raman data: dispersive versus Fourier transform, wavelength calibration, intensity calibration, and in particular the frequency of the excitation laser. While concerns about fluorescence arise in the visible or ultraviolet, most modern (portable) systems use near-infrared excitation lasers, and many of these are relatively close in wavelength. We have investigated the possibility of porting reference data sets from one NIR wavelength system to another: We have constructed a reference library consisting of 145 spectra, including 20 explosives, as well as sundry other compounds and materials using a $1064 \mathrm{~nm}$ spectrometer. These data were used as a reference library to evaluate the same 145 compounds whose experimental spectra were recorded using a second $785 \mathrm{~nm}$ spectrometer. In 128 cases of 145 (or $88.3 \%$ including 20/20 for the explosives), the compounds were correctly identified with a mean "hit score" of 954 of 1000 . Adding in criteria for when to declare a correct match versus when to declare uncertainty, the approach was able to correctly categorize 134 out of 145 spectra, giving a $92.4 \%$ accuracy. For the few that were incorrectly identified, either the matched spectra were spectroscopically similar to the target or the $785 \mathrm{~nm}$ signal was degraded due to fluorescence. The results indicate that imported data recorded at a different NIR wavelength can be successfully used as reference libraries, but key issues must be addressed: the reference data must be of equal or higher resolution than the resolution of the current sensor, the systems require rigorous wavelength calibration, and wavelength-dependent intensity response should be accounted for in the different systems.
\end{abstract}

\section{Introduction}

There are many spectroscopic methods that have decreased the size and cost of the instruments and simultaneously increased their detection potential so as to become routine methods for chemical detection: infrared spectroscopy [1$3]$, visible-NIR reflectance [4], terahertz methods [5, 6], and others. Recently Raman spectroscopy has followed this trend and evolved from a cumbersome method of chemical physics to a common forensic technique [7]. There are currently tens of thousands of deployed spectrometers, many on-line or at-line, and in recent years there has been an advent of portable, even handheld, systems $[8,9]$, as well as remote sensing devices $[10,11]$. Such capabilities make Raman an especially attractive method in the security arena, where substances such as radioactive materials, explosives, and chemical-/biological-warfare agents are a danger not only on the battlefield, but now also to civilian targets. For explosives, detection at several tens of meters has now been demonstrated [12-17] as has Raman chemical imaging of trace explosives, for example, in fingerprints [18]. One implementation of the technique that has really stood out in recent years is Raman spectroscopy using near-infrared lasers $[19,20]$. Such systems have become less cumbersome, inexpensive and still retain the specificity of Raman or IR spectroscopy; namely, they have the ability to detect thousands of different chemicals and substances [21-23].

Contemporary red and near-infrared lasers, particularly the diode lasers, offer great advantages in terms of economy, size, and ruggedness. Should more laser systems be realized at 
wavelengths longer than ca. $1400 \mathrm{~nm}$, enormous advantages could also be realized in terms of eye safety, as the human cornea blocks wavelengths longer than $1400 \mathrm{~nm}$ [24]. At the near-infrared (NIR) wavelengths used in most modern portable systems, most species do not undergo an electronic transition, and thus, empirically, most molecules do not fluoresce or undergo resonance enhancement. The possibility thus exists to port reference data with good success, especially if one considers only NIR excitation wavelengths. But questions arise as to the transferability of Raman data: dispersive versus Fourier transform, wavelength calibration, intensity calibration, and in particular the frequency of the excitation laser [25-27]. Developing such databases is resource-intensive and typically the generated data are not transferred to other instruments. If the reference data were truly portable; however, it obviates the time-consuming and expensive process of re-recording instrument-specific spectral libraries. It may be that data will not be portable to all systems, for example, surface-probing systems or those which use ultraviolet lasers, since different Raman modes can be enhanced differently. However, with red or nearinfrared excitation there is a greatly reduced likelihood of enhancement, fluorescence, or other perturbing phenomena; we have therefore investigated the possibility of porting reference data from one NIR system to another.

While not all aspects can be addressed, this paper considers the possibility of porting an entire database of spectra (i.e., a spectral library of a wide range of substances) recorded at one wavelength $(1064 \mathrm{~nm})$ and employing them for analysis at another wavelength $(785 \mathrm{~nm})$. A companion paper has been submitted [8] that discusses the specifics of adapting such high-resolution spectra to a modern handheld dispersive Raman system. We assume that such a demonstrated portability would be reflected amongst other systems such as those that use extant near-IR lasers (e.g., $633,785,830,980,1047$, and $1064 \mathrm{~nm}$ ), but could also be potentially useful for still other systems that may arise in the future, particularly those with laser wavelengths longer than $1400 \mathrm{~nm}$, such as the lasers used in telecommunications (e.g., $1550 \mathrm{~nm}$ ). At these longer wavelengths not only is there greater eye safety, but also less dispersion from atmospheric particles (aerosol Mie scattering), resulting in better transmission for obvious standoff applications such as explosives [13, 28]. We demonstrate this portability by testing a Raman spectra database of 145 chemicals, including 20 known explosives. To accomplish this, key efforts are made to calibrate on both the wavelength- and intensity-axes such that, in theory, the spectra from any one system will simply overlay those from another.

\section{Experimental and Calibration}

2.1. General Experimental. For these experiments, the spectra were measured using two different Fourier-transform (FT) Raman spectrometers, one using $1064 \mathrm{~nm}$ excitation, the other using $785 \mathrm{~nm}$ excitation. In many instances researchers wish to port data between systems, including between FT and dispersive systems. The merits of FT-spectrometers versus dispersive-instruments for specific applications can be deliberated elsewhere; this paper focuses only on the portability of the data. For both FT instruments [29] and for the dispersive instruments (especially smaller units) frequent and accurate wavelength calibration is paramount $[30,31]$. For this study, it is important to note that while both are FT instruments from the same manufacturer, the instruments are of different wavelength (785 and $1064 \mathrm{~nm}$ ) and have different étendues (numerical aperture), different interferometers (linear air-bearing versus mechanical flex-pivot), different detectors (though both are of the same type, power detection as opposed to photon counting), and differing optical components [7, 22]. Both instruments have a dedicated sample compartment, with either 90- or 180-degree backscatter spectral light collection; for the present work, the $180^{\circ}$ configuration was used including a mirror behind the quartz cuvette for liquid samples. For solids, typically $\sim 5 \mathrm{mg}$ of samples was pressed into an Al sample cup for use with the $180^{\circ}$ collection geometry. While wavelength calibration is critical for any Raman system, the absolute calibration in general can follow more easily for an FT instrument (the Connes advantage) [32]. The intensity calibration for most Raman systems, including the present ones, is not an absolute photometric calibration, but only removes the wavelength-dependent response of the optical system, which arises largely from the detector.

For the $1064 \mathrm{~nm}$ data, the spectra of the neat liquids and solids were recorded using a Bruker FRA 106 Raman module coupled to the interferometer of a Bruker IFS 66v/S vacuum Fourier infrared spectrometer used in purged mode $[33,34]$. The $66 \mathrm{v}$ interferometer [35] used a germanium on calcium fluoride beamsplitter and a white light source for initial interferometer alignment. The FRA 106 Raman accessory consists of a CW $1064 \mathrm{~nm} \mathrm{Nd:YAG} \mathrm{laser} \mathrm{with}$ a focused or unfocused laser spot $[19,36]$ and a notch filter after the F/1 collection lens that removes the Rayleigh scattered light. A $6.0 \mathrm{~mm}$ manual aperture $[37,38]$ was added before the interferometer to achieve the specified $2.0 \mathrm{~cm}^{-1}$ spectral resolution [37, 39]. The Raman-scattered light is modulated by the FTIR interferometer and focused onto a liquid-nitrogen cooled Ge detector inside the 106 accessory.

For the $785 \mathrm{~nm}$ data, a dual wavelength dedicated FTRaman instrument, the Bruker MultiRam 27, was used in the $785 \mathrm{~nm}$ configuration. The MultiRam is a dedicated FT-Raman instrument, but instead of a linear air-bearing interferometer, it has a flex-pivot mechanical bearing system. The MultiRam 27 interferometer is a faster optic f/2.9 versus the f/4.5 of the IFS $66 \mathrm{v}$, so the MultiRam was used with an internal aperture setting of $3.5 \mathrm{~mm}$. The MultiRam beamsplitter is silicon coated onto quartz, and the Raman detector for the 785 channel is a thermo-electrically cooled avalanche pin-diode detector. The MultiRam also uses a proprietary notch filter to remove the Rayleigh line before the interferometer [36]. Both the 1064 and the $785 \mathrm{~nm}$ systems were equipped with a (custom) Stokes/anti-Stokes filter that passes light on both the low- and high-frequency side of the notch frequency.

All data were recorded at $2.0 \mathrm{~cm}^{-1}$ resolution, collecting the $180^{\circ}$-backscattered light. The excitation laser power 
was varied as needed, but seldom exceeded $500 \mathrm{~mW}$. Typically between 300 and 2000 interferograms were averaged, depending on signal quality. Data were collected using forward-backward double-sided interferograms and (due to the broad interferograms) phase-corrected using the power method. To achieve the data portability it is clear that the data must be calibrated in two dimensions, namely (1) the wavelength and (2) the intensity axes. For any given sample, the spectrally corrected plots should overlay one another if done correctly and in absence of any wavelength-dependent phenomena. Extensive calibration for wavelength and intensity proceeded as described below. Most chemicals were purchased as the highest purity available from common chemical suppliers, mostly Sigma-Aldrich. The mineral specimens were from the "Rocks and Minerals of Canada collection" from American Educational Products. The data were only collected after the instrument was wavelength calibrated. The intensity calibration scales the relative response on the $y$-axis as a function of wavelength (described below) and arbitrarily sets the strongest peak to a value of 2.0 with the minimum set to 0.0 .

2.2. Wavelength Calibration. For a Fourier-transform Raman system, frequency calibration is not trivial as it requires an absolute calibration of both (1) the scattered light recording system and (2) the instantaneous (e.g., shot to shot) or averaged frequency of the Raman excitation laser, typically after some stabilization period. To calibrate the interferometer there are several options such as using the narrow absorption lines of a gas such as $\mathrm{CO}$ or $\mathrm{N}_{2} \mathrm{O}$ at low pressure [34, 37]. For both the present spectrometers the interferometer's wavelengths are referenced to those of the HeNe laser, as is common in commercial FT systems. Originally these were calibrated in the near-IR by using a white light source in the sample compartment and observing the $2 \leftarrow 0$ overtone transition of neat carbon monoxide $[34,40]$ in a $10 \mathrm{~cm}$ cell. The transition frequencies were fit by adjusting the $\mathrm{HeNe}$ reference frequency. Using unapodized spectra, a series of CO lines near $4300 \mathrm{~cm}^{-1}$ in the first overtone band were used to match the reported frequencies of Maki and Wells [40]. This proved to be somewhat cumbersome as to where to locate the gas cell, as the interferometer path has few unobstructed beam paths $>10 \mathrm{~cm}$. Recognizing other alternatives, such as an atomic lamp calibration standard, [29] it was found more expedient for reliable calibration of the interferometer to use an array of $\mathrm{Hg}$ lines [41] by bleeding in small amounts of light from the overhead fluorescent lamps. A typical emission spectrum of such lines can be seen in Figure 1. The method is preferred to the $\mathrm{CO}$ absorption technique as the lamp emission light follows exactly the same optical train as the Raman scattered light, and because the Hg-lines method is easier to implement. Using the five $\mathrm{Hg}$ lines depicted in Figure 1 along with the NIST (air) frequencies, the interferometer frequency is calibrated such that the sum of the deviations (obs.-ref.) for all five lines is $\leq$ $0.1 \mathrm{~cm}^{-1}$ on a daily basis. Typically, the average deviation for any given line is $< \pm 0.1 \mathrm{~cm}^{-1}$.

For an FT-Raman system, calibration of the interferometer frequencies alone is not sufficient. The absolute

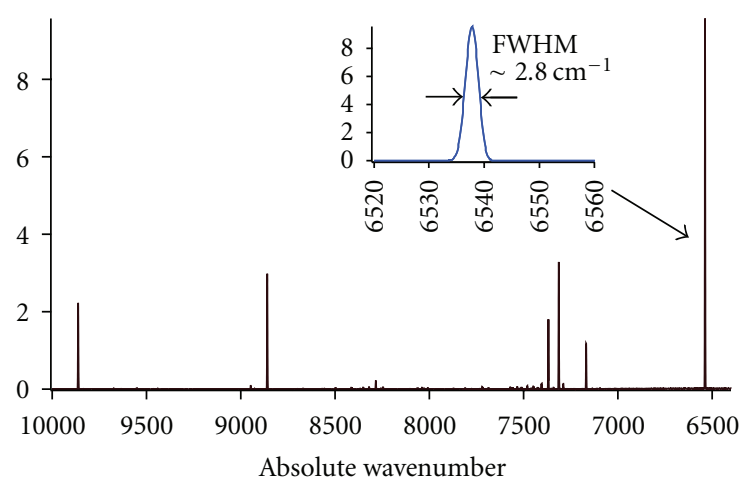

Figure 1: Hg-line spectrum used for interferometer calibration. Several lines are used to calibrate the absolute wavelength response of the interferometer. The Raman laser frequency $\sim 9397 \mathrm{~cm}^{-1}$ is bracketed by the $\mathrm{Hg}$ lines. The inset shows the linewidth (FWHM) of one of the $\mathrm{Hg}$ lines.

wavelength of the continuous wave excitation laser must also be set. There are methods to achieve this including using absolute Raman wavelength standards such as cyclohexane $\mathrm{C}_{6} \mathrm{H}_{12}$ frequency shifts $[25,31,42-44]$. If using just one or two such transitions, however, it is possible as a happenstance that a small frequency offset of the Raman laser could be equally offset by a small miscalibration of the interferometer frequency in the opposite direction. This can be especially true for certain NIR solid state lasers with strong room temperature frequency fluctuation. In the present system we have opted to use a laser line notch filter that passes both the Stokes and anti-Stokes lines. In this method the calibration of the excitation laser is accomplished by setting the software value of the Raman laser frequency such that the absolute values of the Stokes and anti-Stokes frequency shifts for a given vibrational mode are made equivalent. If the input value of the excitation laser frequency were incorrect, the nominal Raman "zero" frequency position would be shifted, resulting in differing Stokes and anti-Stokes frequencies. For the Raman Nd:YAG $1064 \mathrm{~nm}$ laser systems, a daily Stokes versus anti-Stokes calibration is performed using a strong scatterer, typically elemental sulfur $[19,38]$. Low frequency modes are advantageous here as the detector responsivity is limited to approximately $-1,000 \mathrm{~cm}^{-1}$ anti-Stokes shift (detector's bandgap or work function cutoff). The $785 \mathrm{~nm}$ laser wavelength was calibrated using $785 \mathrm{~nm} \mathrm{KClO}_{3}$ Raman spectra against the $\mathrm{KClO}_{3}$ spectra obtained from Stokes and anti-Stokes calibrated $1064 \mathrm{~nm}$ system. For most species, however, the sample itself can be employed to calibrate the Raman laser frequency as seen in Figure 2 for $\mathrm{KMnO}_{4}$. The $\mathrm{KMnO}_{4}$ provided several low frequency lines with which one can obtain calibration accuracies of the Stokes and antiStokes Raman shifts of better than $0.2 \mathrm{~cm}^{-1}$ using a few of the strongest lines. For both the $1064 \mathrm{~nm} \mathrm{Nd:YAG} \mathrm{laser} \mathrm{and}$ the $785 \mathrm{~nm}$ GaAlAs laser, the nominal frequency was adjusted until the agreement was within $\pm 0.20 \mathrm{~cm}^{-1}$. Due to the inherent Raman laser linewidths, typically 1.0 to $1.5 \mathrm{~cm}^{-1}$, greater precision is not warranted. 


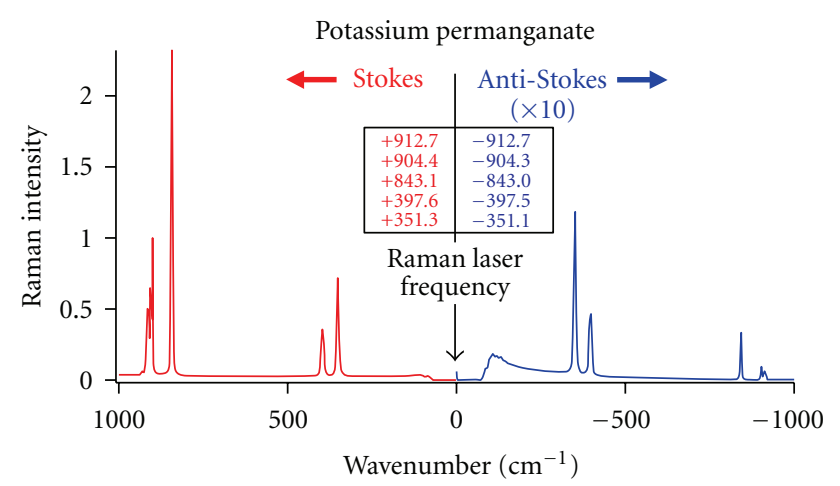

FIgURE 2: Calibration scheme demonstrating how Stokes and antiStokes lines are used to calibrate the laser frequency such that the Stokes lines are at the same shift with the opposite sign of the anti-Stokes lines. The present data show Raman lines of potassium permanganate. Typically the values are $\leq 0.20 \mathrm{~cm}^{-1}$ absolute value of each other. The excitation laser line is nominally at $9397.58 \mathrm{~cm}^{-1}$ (air) absolute frequency, but has typical daily variations of $\pm 0.50 \mathrm{~cm}^{-1}$ or more.

2.3. Intensity Calibration. In some instances, intensity calibration plays a lesser role as chemical identification is accomplished only via peak look-up tables. In many cases, however, the relative amplitude of the peaks plays a key role as peak heights as well as positions are used for identification. This is more crucial when methods such as partial least squares are used for chemometric analysis [45]. In some cases absolute scattering coefficients are obtained, and in most cases this is done by comparison to an absolute standard [7]. As Ray and McCreery have pointed out, [27] obtaining Raman intensity values that are corrected even on a relative scale, that is, that properly ratio out the instrumental response, is more challenging than first supposed. The challenge lies in the fact that the Raman experiment is a single-beam experiment; that is, it is not a self-ratioing measurement such as an infrared transmittance measurement.

In our earlier measurements, the calibration of the scattering intensity for its wavelength dependence was accomplished using a broadband source of known brightness [46]. Other workers have accomplished this using calibrated blackbodies whose absolute emittance was known as a function of drive current. However, the FRA 106 Raman module has a $3000 \mathrm{~K}$ white light source whose intensity is scattered into the spectrometer off a NIST-traceable Spectralon surface mounted at the sample position. The instrument response function as a function of wavelength was generated by ratioing this spectrum relative to the intensity predicted by Planck's law for a $3000 \mathrm{~K}$ source. The quality of the intensity correction was assessed by measuring the intensities in the corrected Stokes and anti-Stokes spectra; the relative intensities were within a few percent of the expected value [21] and the agreement was good.

While a useful method, this is not especially practical for field instruments. Recently, the National Institute of Standards and Technology [25] has developed standard calibrated targets SRM 2241 and SRM2244, for use with either 785 or $1064 \mathrm{~nm}$ excitation lasers, respectively. By measuring the spectrum of such a target and comparing it to the NIST-calibrated spectrum we are able to determine a calibrated system response curve from 3500 to $100 \mathrm{~cm}^{-1}$. After determining the system response in this manner all that is required for intensity calibration is a simple spectral multiplication. Subsequent spectra are then multiplied by this frequency-dependent $y$-axis scaling factor.

An example of the relative response factor for our $1064 \mathrm{~nm}$ system between +3500 to $+100 \mathrm{~cm}^{-1}$ can be seen in Figure 3, as well as a Raman spectrum of ortho-xylene before and after the correction factor has been applied. It is seen that the intensity correction is changing the most rapidly at the ends of the spectrum near the system notch filter cutoff and at the red end of the spectrum $\left(\Delta v=3500 \mathrm{~cm}^{-1}, v \sim 5900 \mathrm{~cm}^{-1}\right)$ where the Ge detector is approaching its bandgap cutoff. The intensity correction results using the two different methods were found to give comparable results.

2.4. Molecule Selection. To test the library's portability a list of species was derived. The list of the composite library contains a total of 145 molecules. Of these, 20 are known explosive materials specifically selected and these are summarized with some of their properties in Table 1. For the nonexplosive materials we intentionally chose several different categories and colors of molecules and materials so as to attain a good cross-representation. While a more extensive list is always desirable for statistical purposes, time and budget constraints would not allow this. Many of the reference materials are pure chemicals commonly found in a modern laboratory, but care was taken such that the list represented a good variety of chemical classes, rather than just a few classes such as, for example, alkanes, alkenes, and alcohols. The composite list was designed to contain at least three species from each of the several chemical categories found in Table 2: aldehydes, alkenes, amines, amino acids, aromatics, carboxylic acids, halogenated organics, ketones, thiols, mineral acids, ionic simple salts, and organometallics. Table 2 characterizes the chemical breakdown of these 125 non-explosive materials. For those pure chemical species with two or more functional groups, the compound could be counted in two different categories; for example, 1, 3dichlorobenzene would be tallied both as an aromatic and as a halogenated organic. Besides pure chemicals found in a chemical laboratory, the list was further expanded to contain common household and industrial materials, and also materials such as mineral acids, common minerals and some biological samples. Some of the common materials included items such as wood, concrete, minerals, and dirt; plastics such as nylon, Teflon, and a water bottle; other fabrics such as wool, cotton, leather; and household materials.

The list was thus completed and expanded to include a total spectral database of 145 compounds and materials including the 20 explosives as described above-with a significant effort to derive a representative potpourri of different chemical classes. What is only partially indicated is that several of the non-explosive chemical samples were 


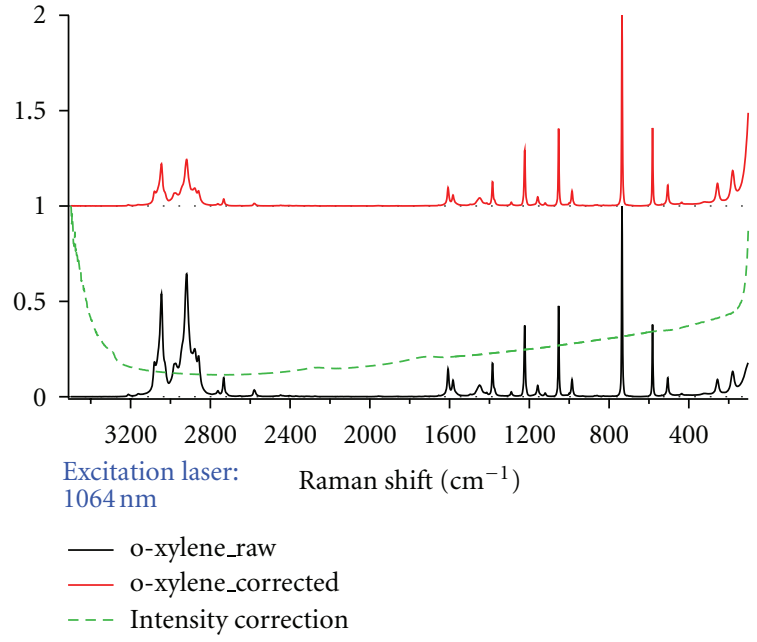

Figure 3: Response of the $1064 \mathrm{~nm}$ FT-Raman system before and after correcting for the spectrometer intensity response function using the NIST 2244 standard plotted as a function of Raman shift. The $\mathrm{x}$-axis represents 5897 to $9327 \mathrm{~cm}^{-1}$ absolute wavenumbers, with the intensity correction factor generated by the standard plotted as the dashed green line. The "raw" spectrum is plotted in black as the lower trace, the corrected spectrum in red as the upper trace. These spectra are arbitrarily scaled 0 to 1 on the ordinate and have been vertically offset for clarity.

intentionally selected due to their mixture of light and dark materials, that is, their physical colors. While there was a long list of clear liquids and white solids (the "unknown white powder" challenge), there were also many deeply colored materials, which are more susceptible to fluorescence at $785 \mathrm{~nm}$. To further ensure a representative cross-section, specific examples were also intentionally chosen because they are known to fluoresce, in some cases more strongly at 785 than $1064 \mathrm{~nm}$. These include species such as calcium hypochlorite, but also some ink dyes as suggested by the work of Geiman et al. [47], namely, acid red 52 dye and crystal violet dye. The complete list of chemical species and breakdown by chemical category is available as Supplementary material available online at doi:10.1155/2012/297056, with the classes summarized in Table 2.

\section{Results and Discussion: Spectroscopy}

As discussed above, the intensity calibration scales only the relative (wavelength-dependent) response as seen in Figure 3, with the spectrum maximum arbitrarily set to a value of 2.0. Absolute cross-sections are not needed for this study as the goal is only for identification not quantification. That is to say, the present results do not explicitly account for the " $v^{4}$ " factor associated with excitation wavelength. Strictly speaking, if a power-detecting element is used (common in NIR systems), the $\left(\nu_{0}-v_{k}\right)^{4}$ expression is appropriate whereas if a photon-counting device (common in visible Raman systems) is used, the relative response term is $\nu_{0}\left(\nu_{0}-v_{k}\right)^{3}$, where $\nu_{0}$ is the laser frequency and $\nu_{k}$ the vibrational mode frequency [22]. As has been noted before

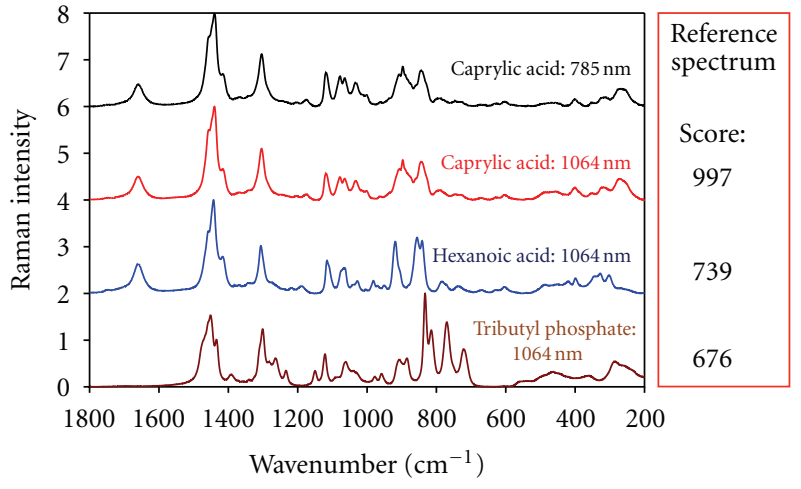

FIgURE 4: Example of a successful identification for comparing the $785 \mathrm{~nm}$ data versus the $1064 \mathrm{~nm}$ library. The result is typical; for most species the $1064 \mathrm{~nm}$ spectrum of the same compound is identified as the top candidate "hit" for the 785 spectrum. Scores for the top three hits are at right. Spectra are scaled 0 to 2 and have been vertically offset for clarity.

$[7,22]$ any measured intensity of a Raman band in fact has contributions from 3 sources, namely, (1) the Raman activity itself that is intrinsic to the scattering species; this is the term that is typically calculated by theoretical models, (2) a statistical mechanical term accounting for temperature (which affects the population of the scattering vibrational state), and (3) the well-known " $\nu^{4}$ " factor that depends on the laser excitation frequency and the type of photometric detection in the Raman spectrometer as discussed above.

The quality of the derived spectra is very high due to the laboratory nature of our experiments with adequate averaging time. After proper wavelength and (relative) intensity calibration, and barring any substantial fluorescence or resonance effects, the spectra from the differing wavelength systems should nearly superimpose on one another. In almost all cases our results have shown this to be the case. The 1064 reference data can readily serve as reference spectra for 785 systems, as shown for the two FT-Raman systems shown here, as well as for dispersive 785 handheld Raman systems [8]. This is seen for a typical example in Figure 4 and was the case for almost all measured species. The search algorithm and criteria for declaring a match are described below, but Figure 4 illustrates the results of this process for caprylic acid. In particular, caprylic acid (octanoic acid) is a "785 clean spectrum" so the search clearly identifies the correct compound at 1064 as the top hit. The traces of the "search" 785 spectrum and the "reference" 1064 spectrum are seen as the top two traces in the plot and are identical to the eye. The second hit, with a relatively high score, is the chemically and spectroscopically similar hexanoic acid, with tributyl phosphate detected as the third hit.

For the explosives, the commercial grade materials such as those used for mining and demolition are sold by trade names and generally consist of a mixture of energetic materials. The variety and relative percent concentrations of the materials used vary widely and depend upon required performance and cost. Many of the commercial products, while sold under different names, have some strong commonalities 
TABLE 1: Summary of the 20 explosives measured by both the 785 and $1064 \mathrm{~nm}$ Raman systems. In several cases the materials are named by their primary chemical component(s), followed by the common or commercial name, since the primary components define the spectrum. AN: ammonium nitrate, EGDN: ethylene glycol dinitrate, PETN: Pentaerythritol tetranitrate, NC: nitrocellulose, NG: nitroglycerine.

\begin{tabular}{|c|c|c|}
\hline Sample & Name & Comments/chemical composition \\
\hline 1 & $\mathrm{AN} / \mathrm{NaNO}_{3}$-Coalmex & Often contains $\mathrm{AN}, \mathrm{NaNO}_{3}, \mathrm{EGDN}$ \\
\hline 2 & $\mathrm{AN} / \mathrm{NaNO}_{3}$-Dyno AP & Emulsion explosive for mining, $\mathrm{AN}, \mathrm{NaNO}_{3}$ \\
\hline 3 & $\mathrm{AN} / \mathrm{NaNO}_{3}$-Dynomax Pro & Dynamite blasting gelatine, NG, EGDN, NC, $\mathrm{AN}, \mathrm{NaNO}_{3}$ \\
\hline 4 & $\mathrm{AN} / \mathrm{NaNO}_{3}$-Powerfrac & Often contains AN, $\mathrm{NaNO}_{3}, \mathrm{EGDN}, \mathrm{S}, \mathrm{NG}, \mathrm{NC}$ \\
\hline 5 & $\mathrm{AN} / \mathrm{NaNO}_{3}$-Red-D Gel-B & Dynamite- often contains NG, EGDN, NC, AN, $\mathrm{NaNO}_{3}$ \\
\hline 6 & HMX & Octahydro-1,3,5,7-tetranitro-1,3,5,7-tetrazocine \\
\hline 7 & Nitroglycerine & NG, Liquid, 1,2,3-Trinitroxypropane \\
\hline 8 & Nitroguanidine & $\mathrm{CH}_{4} \mathrm{~N}_{4} \mathrm{O}_{2}$, used in powders to reduce barrel erosion \\
\hline 9 & PETN & Pentaerythritol tetranitrate \\
\hline 10 & PETN-Primasheet 1000 & PETN-65\% based rubberized explosive \\
\hline 11 & PETN-Semtex 10 & Largely contains $\sim 85 \%$ PETN \\
\hline 12 & PETN-Semtex A & Largely contains $\sim 84 \%$ PETN \\
\hline 13 & PETN-Semtex H & Often contains $>25 \%$ PETN, $<65 \%$ RDX \\
\hline 14 & Picric acid & 2,4,6-Trinitrophenol, forms sensitive metal salts \\
\hline 15 & RDX & 1,3,5-Trinitroperhydro-1,3,5-triazine \\
\hline 16 & RDX-C4 & Contains $\sim 91 \%$ RDX plus plasticizers and binders \\
\hline 17 & RDX-PE4 & Similar to C-4, an RDX-based explosive (white) \\
\hline 18 & RDX-PW4 & Largely RDX-based plastic explosive \\
\hline 19 & TNB & Trinitrobenzene, commercial mining high explosive \\
\hline 20 & TNT & 2,4,6-Trinitrotoluene \\
\hline
\end{tabular}

TABLE 2: Chemical and material classes of the 125 nonexplosive chemicals and materials that are amongst the species that were tested. For the pure chemicals, species with multiple functional groups were tabulated in each class.

\begin{tabular}{lclc}
\hline Class/type of compound & $\begin{array}{c}\text { Number of compounds in class } \\
\text { Organic classes }\end{array}$ & Class/type of compound & Number of compounds in class \\
Inorganic classes
\end{tabular}

in the material components. For example, many mining materials contain various levels of ammonium nitrate and sodium nitrate along with other materials. Due to the strong Raman activity of the $\mathrm{NO}_{3}^{-}$moiety, both $\mathrm{NH}_{4} \mathrm{NO}_{3}(\mathrm{AN})$ and $\mathrm{NaNO}_{3}$ provide features that dominate the spectrum, while the smaller features help aide slightly in the identification of the specific formulation. Thus, the explosive materials listed in Table 1 are segregated and categorized based upon their primary chemical components or compound groups. Subtle differences due to noise and baseline correction can 
lead to slight changes in relative peak intensity and even peak position. Due to the similar makeup of several of the explosives, such differences were likely the cause of the top hit of an analyte not being an exact match to that formulation but the top hit was for a formulation containing nearly identical chemical components to those in the analyte. When the library generated at $1064 \mathrm{~nm}$ was used for identifying samples measured at $785 \mathrm{~nm}$ and was based upon selecting the correct compound group (e.g., if any of the $\mathrm{AN} / \mathrm{NaNO}_{3}$ formulations were identified), all 20 explosive formulations in Table 1 were correctly identified.

There were a few outliers in the statistical data, a few of which merit additional explanation: for a few species, the fluorescence phenomenon stimulated by the excitation laser light generated a signal that is more intense than the light scattered by the weaker Raman effect; this was true for those species selected due to their $785 \mathrm{~nm}$ fluorescence [47]. In fluorescence, the excitation light is absorbed and re-emitted via an electronic transition that does not involve a change in the spin quantum number; at room temperature the fluorescence bands typically manifest themselves as a broad and featureless background. In some cases, this fluorescence background signal can be removed via baseline correction, but many researchers have shown that shifting to longer wavelength excitation is even more advantageous, as Lewis et al. have shown for certain explosives [48, 49]. In the present studies a broad fluorescence was observed in $\sim 10$ out of 145 cases, but identification was still possible for a few of these materials. Typically the method used in most sensors is to simply filter the analysis via baseline correction such as with a rolling circle filter or some other baseline correction algorithm. While the phenomena are real optical phenomena, they are usually featureless and are simply removed as backgrounds via a software algorithm, leaving only the Raman peaks.

Interestingly, there were also a few anomalous situations where there was significant spectral change (not just a fluorescence background) depending upon the excitation wavelength. One example, illustrated in Figure 5, was for neat $\mathrm{Er}_{2} \mathrm{O}_{3}$. While a few peaks are common and are likely Raman bands, it appears that either the $1064 \mathrm{~nm}$ or $785 \mathrm{~nm}$ excitation, or both, has excited transitions into low-lying electronic states. The resulting fluorescence then dominates the emission spectrum. In this particular case the fluorescence(s) are not broad and featureless as in the other cases, but are structured and are likely associated with the pseudoatomic transitions of the $\mathrm{Er}^{3+}$ cation. The translanthanides and trans-actinides typically have multiple low-lying electronic states that give the species their colors. In their studies of $\mathrm{Er}_{2} \mathrm{O}_{3}$ nanocolloids, Patel et al. [50] discuss an upconversion signal observed at $740 \mathrm{~nm}$ when pumping with a $980 \mathrm{~nm}$ multimode diode laser that is resonant with ${ }^{4} \mathrm{I}_{11 / 2}$ state. This was ascribed to a two photon excitation processes of the type ${ }^{4} \mathrm{I}_{15 / 2} \rightarrow{ }^{4} \mathrm{I}_{11 / 2}$ and ${ }^{4} \mathrm{I}_{13 / 2} \rightarrow{ }^{4} \mathrm{~F}_{9 / 2}$ that resulted (after internal conversion) to a ${ }^{4} \mathrm{I}_{9 / 2} \rightarrow{ }^{4} \mathrm{I}_{15 / 2}$ emitted. The structured bands we see in the $785 \mathrm{~nm}$ Raman spectrum near $2500 \mathrm{~cm}^{-1}$ shift correspond to $10,235 \mathrm{~cm}^{-1}$ absolute or about $977 \mathrm{~nm}$ and are likely emission from the original state into which their $980 \mathrm{~nm}$ laser pumped, that is,

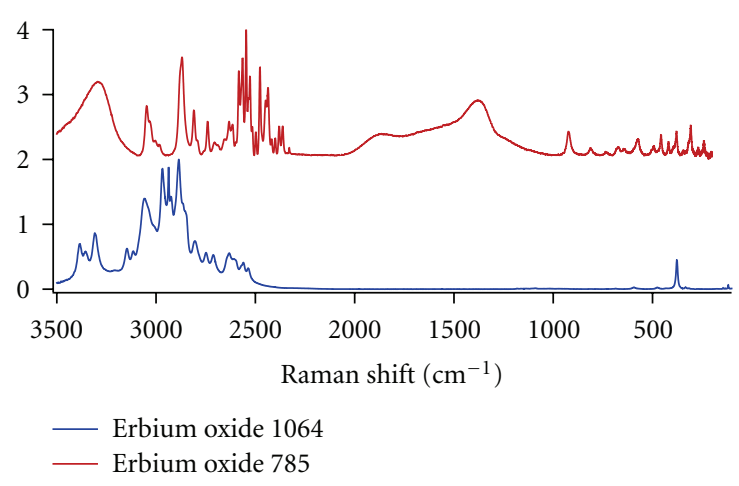

FIGURE 5: Results of the $\mathrm{Er}_{2} \mathrm{O}_{3}$ spectra at the two different excitation wavelengths. The spectra have been vertically offset for clarity with $y$-axis scaled $0-2$. The results are discussed in the text.

an ${ }^{4} \mathrm{I}_{11 / 2} \rightarrow{ }^{4} \mathrm{I}_{15 / 2}$ emission, though further measurements would be needed to confirm this [51].

\section{Results and Discussion: Statistical Analysis}

For the analysis, our overarching goal was to determine whether a spectrum recorded using $785 \mathrm{~nm}$ excitation can be successfully identified using a library created from $1064 \mathrm{~nm}$ reference data. To test the hypothesis that the $785 \mathrm{~nm}$ and $1064 \mathrm{~nm}$ spectra are interchangeable after wavelength and intensity corrections, a comparison study was conducted, where the Bruker OPUS commercial spectral searching software was used and the $785 \mathrm{~nm}$ intensity-corrected spectra were treated as test cases and searched against a $1064 \mathrm{~nm}$ intensity-corrected library. The software has several different search algorithms, each with several possible variations to it. However, our intent was not to perform a comprehensive study of different algorithms, but rather to measure the similarity of the $785 \mathrm{~nm}$ and $1064 \mathrm{~nm}$ spectra under ordinary laboratory conditions. Therefore, the OPUS basic search algorithm was used.

The OPUS basic search algorithm works by matching peaks without regard to their intensities. In particular, for any given spectrum, a peak table is constructed. This peak table is used to create a signature spectrum consisting of Lorentz curves for every peak, each of which has a height of one. To compare a test case to a library compound, the algorithm looks up all of the test signature peaks in the library signature. Those test peaks that appear in the library signature are assigned a value of one, whereas those that are different or do not appear in the library signature are assigned a lesser value, with a minimum of zero. A hit quality is then calculated as the average of these values times 1000 . More simply, when all test case peaks are found in given library signature spectrum, the hit quality is 1000 . When none of them are found, the hit quality is zero. For example, when three of four test signature peaks are found in a given library entry, the hit quality is $3 / 4 * 1000=750$. For a given test case, this comparison process is repeated for every entry 
of the spectral library, and the three library compounds with the highest hit quality - the top three hits-are reported.

OPUS allows for some parameter adjustment in its basic search algorithm. In particular, there is an adjustable "search sensitivity" parameter. While specific details about this parameter are unavailable, its value, an integer between 1 and 20, relates to the sensitivity with which OPUS identifies peaks, 20 dictating a much closer match. Loosely interpreted, a higher search sensitivity means the peak locations must be more precise for the algorithm to give a higher hit score, to the point that Bruker did not recommend a setting of 15 or higher for this application. The search sensitivity also affects the number of peaks created in the temporary peak table for the spectrum being searched. Thus, a higher search sensitivity implies a stronger match and implies more peaks may have been created and matched than a lower sensitivity value. OPUS suggests testing different values to determine which is best for a particular application. For our application, a search sensitivity of 7 was chosen because preliminary results showed this value tended to generate favorable search results in terms of good discrimination.

A $1064 \mathrm{~nm}$ library was created in OPUS using all 145 compound spectra. The $785 \mathrm{~nm}$ spectra were then individually loaded and searched against the $1064 \mathrm{~nm}$ library via the basic search algorithm. In terms of the explosives, using the chemical formulation grouping, all 20 explosives were correctly identified. It should also be noted that for many of these formulations the top 3 or 4 hits were for similar compounds and all had very similar high scores. Thus the explosive materials listed in Table 1 are segregated and categorized based upon primary chemical components or compound groups. When the library generated at $1064 \mathrm{~nm}$ was used for identifying samples measured at $785 \mathrm{~nm}$ and was based upon selecting the correct compound group, all 20 explosive formulations were correctly identified. We also note that, for the explosives, the first hit minus second hit refers to the first hit minus the first hit outside of its compound group. For example, $\mathrm{NH}_{4} \mathrm{NO}_{3}-\mathrm{NaNO}_{3}$-Coalmex has a top hit of 1000. Its second, third, fourth, and fifth hits are also 1000 with each hit belonging to the same compound group. Its first hit outside of the group is potassium nitrate with a hit score of 468 . Therefore the distance between first and second hits for AN-NaNO3 is 532, the difference of 1000 and 468.

While the explosives were all easily identified with the 1064 imported library, for the more general case of relying on an algorithm for identifications, it is important to first establish objective criteria for declaring a match. For example, when the top hit score is near 1000, there is strong evidence for a match, but not so when the top hit score is near 400. Even in the case of a high score, if the top two hit scores are so close as to be virtually identical (say 985 and 988), then there is ambiguity in declaring one or the other a match. A concrete example of this is provided in Figure 6. In the figure, hair gel had petroleum jelly as its top hit with hair gel as its second hit. This implies the search algorithm cannot, for certain, determine which spectrum is the correct match. In this particular case, because the hair gel was determined to be made of almost exclusively petroleum jelly with a few

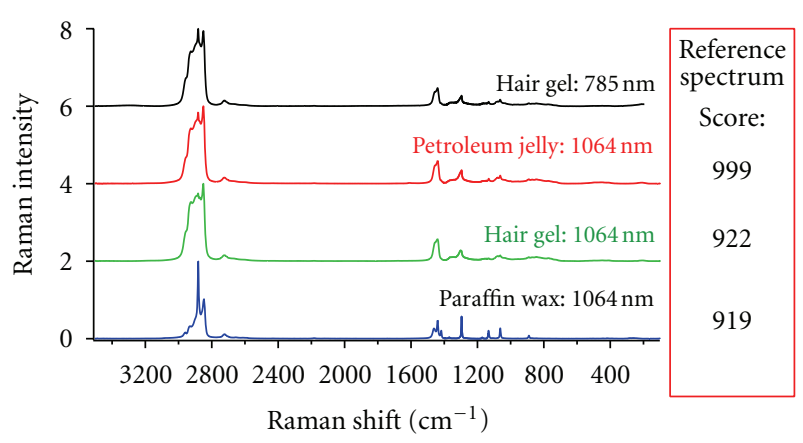

Figure 6: Spectral search example of one of the few false hits showing hair gel at $785 \mathrm{~nm}$ with petroleum jelly (1064) as its top hit and hair gel (1064) as its second hit. The distance between the top two hits is more than 60 (with a distance of 77) and the top hit is above 775 so it is declared a match. These spectra are all scaled $0-2$ on the $y$-axis and have been vertically offset for clarity.

trace additives (namely a coloring agent) the major peaks were therefore in the same positions. Hair gel is an example of a "false hit"; however, in the more general case, a "false miss" is usually caused by peaks in (nearly) the same $x$-axis location but which have slightly different peak intensities or profiles. In such cases a more definitive criterion is needed to declare a match.

Based on the above considerations, two criteria were thus established for matching the $785 \mathrm{~nm}$ spectra to the $1064 \mathrm{~nm}$ library. The first criterion, referred to as the naïve criterion, selects the top hit as a match regardless of the hit score. Using this criterion, 128 out of the 145 spectra at $785 \mathrm{~nm}(88.3 \%)$ were correctly identified. The second method, referred to here as the dual criteria, is designed to take into account not only the top hit but also the difference between the first and second hits. In this case, the top hit must be above a minimum threshold to be declared a match, and the distance between the first and second hit must be larger than a specified value. These dual criteria ensure that (1) the top hit closely matches the test spectrum, and (2) there are no other library spectra similar enough to the test spectrum to cause ambiguity. After initial experimentation, the dual criteria were set so that the minimum hit threshold was 775 and the minimum required distance between the first and second hit was at least 60 . Those test spectra meeting both criteria were declared a match. All others were declared "uncertain."

Figure 7 contains the results of matching the $785 \mathrm{~nm}$ spectra to the $1064 \mathrm{~nm}$ library using the dual criteria. The $y$ axis contains the top hit score and the $x$-axis is the distance between the first two hit scores. The points inside the shaded region are the ones that are declared a match using the dual criteria. The markers indicate whether the corresponding spectrum was a (1) correct match (true hit), (2) an incorrect match (false hit), (3) an incorrect elimination (false miss), or (4) a correct elimination (mismatch excluded).

Table 3 provides a comparison between the results using the naïve criteria and the dual criteria. As can be seen, the number of false matches using the naïve criteria is 17 , and this number drops to 4 using the dual criteria. This large 


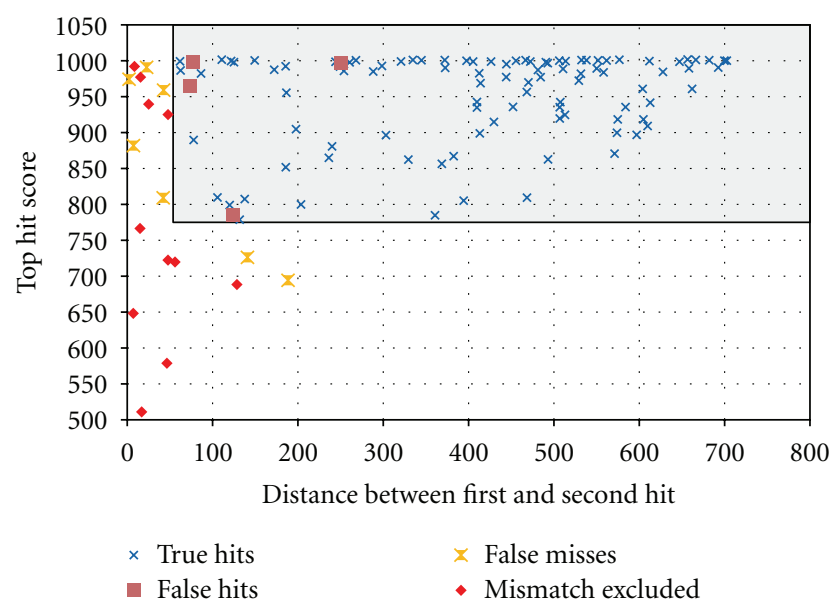

FIGURE 7: $785 \mathrm{~nm}$-to-1064 nm comparison results using the dual criteria with all possible outcomes indicated. The true hits occur when the top hit is the correct spectrum and is declared a match, the false hits occur when a top hit is an incorrect spectrum but is declared a match, the false misses occur when the top hit is the correct spectrum but is declared not a match, and the mismatch excluded occurs when the top hit is an incorrect spectrum and is declared not a match.

decrease in false matches is due to the addition of a new classification category, "uncertain," for those test spectra that do not meet both of the dual criteria. Eleven of the original false matches are moved into the "uncertain" category when the dual criteria are used. Also because of the "uncertain" category, the number of false misses increases when going from the naïve to the dual criteria. In particular, the naïve criterion always declares a match and so the number of false misses must be zero. On the other hand, the dual criteria do not always find a match, and the number of mismatches is 7. Despite the increase in the number of false misses, we note the overall error, the sum of the false matches and false misses, decreases from 17 to 11 when going from the naïve criterion to the dual criteria, suggesting the dual criteria improve accuracy of the identification process.

\section{Summary and Conclusions}

We measured Raman spectra of 145 sundry chemicals, including 20 explosives, representing multiple chemical classes using both $785 \mathrm{~nm}$ and $1064 \mathrm{~nm}$ excitation. This cross-section represented many different chemical classes (e.g., alkanes, alcohols, nitrates, etc.) along with common materials (concrete, sand, wood, etc.). From the measured data, a $1064 \mathrm{~nm}$ library was constructed and the $785 \mathrm{~nm}$ spectra were individually searched against this library to determine the success of searching against reference spectra recorded at a different wavelength. All 20 explosives tested were correctly and readily identified using the 1064 to $785 \mathrm{~nm}$ ported data. Moreover, the initial results indicated that after all searches were complete $88 \%$ of the $785 \mathrm{~nm}$ spectra were correctly identified using the naïve criteria. These results clearly show good discrimination and utility
TABLE 3: Results of spectral searching using the naïve and dual criteria. See text for details.

\begin{tabular}{lcc}
\hline Search criteria & $\begin{array}{c}\text { No. of false matches } \\
(\%)\end{array}$ & $\begin{array}{c}\text { No. of false misses } \\
(\%)\end{array}$ \\
\hline Naïve & $17(11.7 \%)$ & $0(0 \%)$ \\
Dual & $4(2.8 \%)$ & $7(4.8 \%)$ \\
\hline
\end{tabular}

of reference data from a different NIR wavelength system. The use of the "dual" criteria reduced the total number of errors even further, resulting in a $92.4 \%$ correct identification rate. It is again noted that for those $785 \mathrm{~nm}$ cases where fluorescence prevented identification, this would likely have happened regardless of the excitation wavelength of the reference data.

In the near-infrared there are now several dispersive and also Fourier transform spectrometers available. With but a few caveats our results have shown that the data can in fact be ported from one system to another. The data can ideally be used with many common near infrared lasers: $785,830,960$, 1047,1064 or $1550 \mathrm{~nm}$. This is especially true for algorithms that use only relative peak heights or peak positions for identification. For visible or UV systems, it is more important to consider those phenomena that can change relative peak heights of different vibration modes, resonance Raman, fluorescence, and so forth. It is important to note that the reference data must be of equal or higher resolution compared with the target spectra and the spectrometers require rigorous wavelength calibration. Ideally wavelengthdependent intensity response should be accounted for as well.

\section{Acknowledgments}

The research described in this paper was supported by the Combating Terrorism Technical Support Office (CTTSO) and the Joint Improvised Explosive Device Defeat Organization (JIEDDO). The authors thank sponsors for their support. The Pacific Northwest National Laboratory is a multiprogram national laboratory operated by Battelle for the U.S. Department of Energy under Contract DEAC05-76RL01830. Portions of the research were performed using EMSL, a national scientific user facility sponsored by the Department of Energy's Office of Biological and Environmental Research and located at PNNL. We thank Drs. Arno Simon and Juergen Sawatzki of Bruker Optik for discussions about the spectrometers and also Dr. Martin Pirzer for discussions about OPUS software specifics. We also thank Dr. Trish McDaniel, Dr. Lou Wasserzug, and Dr. Peter Hotchkiss for their involvement and support of this research.

\section{References}

[1] I. R. Burling, R. J. Yokelson, S. K. Akagi et al., "Airborne and ground-based measurements of the trace gases and particles emitted by prescribed fires in the United States," Atmospheric Chemistry and Physics, vol. 11, no. 23, pp. 12197-12216, 2011. 
[2] B. E. Bernacki and M. C. Phillips, "Standoff hyperspectral imaging of explosives residues using broadly tunable external cavity quantum cascade laser illumination," in Chemical, Biological, Radiological, Nuclear, and Explosives (CBRNE) Sensing XI, vol. 7665, article 76650I of Proceedings of SPIE, Orlando, Fla , USA, April 2010.

[3] T. J. Johnson, R. L. Sams, S. D. Burton, and T. A. Blake, "Absolute integrated intensities of vapor-phase hydrogen peroxide $\left(\mathrm{H}_{2} \mathrm{O}_{2}\right)$ in the mid-infrared at atmospheric pressure," Analytical and Bioanalytical Chemistry, vol. 395, no. 2, pp. 377-386, 2009.

[4] W. M. Wendlandt and H. G. Hecht, Reflectance Spectroscopy, Interscience Publishers, London, UK, 1966.

[5] T. J. Johnson, N. B. Valentine, and S. W. Sharpe, "Midinfrared versus far-infrared (THz) relative intensities of roomtemperature Bacillus spores," Chemical Physics Letters, vol. 403, no. 1-3, pp. 152-157, 2005.

[6] D. M. Sheen, D. L. McMakin, and T. E. Hall, "Detection of Explosives by Millimeter-wave Imaging," in Counterterrorist Detection Techniques of Explosives, J. Yinon, Ed., pp. 237-278, Elsevier, Amsterdam, The Netherlands, 2007.

[7] R. L. McCreery, "Photometric standards for Raman spectroscopy," in Handbook of Vibrational Spectroscopy, J. M. Chalmers and P. R. Griffiths, Eds., Wiley, New York, NY, USA, 2002.

[8] J. C. Weatherall, J. Barber, C. S. Brauer et al., "Adapting Raman spectra from laboratory spectrometers to portable detection systems," Applied Spectroscopy.

[9] P. Vandenabeele, K. Castro, M. Hargreaves, L. Moens, J. M. Madariaga, and H. G. M. Edwards, "Comparative study of mobile Raman instrumentation for art analysis," Analytica Chimica Acta, vol. 588, no. 1, pp. 108-116, 2007.

[10] N. R. Butt, M. Nilsson, A. Jakobsson et al., "Classification of Raman spectra to detect hidden explosives," IEEE Geoscience and Remote Sensing Letters, vol. 8, no. 3, pp. 517-521, 2011.

[11] A. Ehlerding, I. Johansson, S. Wallin, and H. Östmark, "Electro-optical remote sensing, photonic technologies, and applications IV," vol. 7835, article 783507 of Proceedings of SPIE, Toulouse, France, September 2010.

[12] S. Wallin, A. Pettersson, H. Östmark, and A. Hobro, "Laserbased standoff detection of explosives: a critical review," Analytical and Bioanalytical Chemistry, vol. 395, no. 2, pp. 259-274, 2009.

[13] A. Pettersson, I. Johansson, S. Wallin, M. Nordberg, and H. Östmark, "Near real-time standoff detection of explosives in a realistic outdoor environment at $55 \mathrm{~m}$ distance," Propellants, Explosives, Pyrotechnics, vol. 34, no. 4, pp. 297-306, 2009.

[14] M. D. Ray, A. J. Sedlacek, and M. Wu, "Ultraviolet miniRaman lidar for stand-off, in situ identification of chemical surface contaminants," Review of Scientific Instruments, vol. 71, no. 9, pp. 3485-3489, 2000.

[15] M. Wu, M. Ray, K. H. Fung, M. W. Ruckman, D. Harder, and A. J. Sedlacek, "Stand-off detection of chemicals by UV Raman spectroscopy," Applied Spectroscopy, vol. 54, no. 6, pp. 800806, 2000.

[16] B. Zachhuber, G. Ramer, A. Hobro, E. T. H. Chrysostom, and B. Lendl, "Stand-off Raman spectroscopy: a powerful technique for qualitative and quantitative analysis of inorganic and organic compounds including explosives," Analytical and Bioanalytical Chemistry, vol. 400, no. 8, pp. 2439-2447, 2011.

[17] B. Zachhuber, C. Gasser, E. T. H. Chrysostom, and B. Lendl, "Stand-off Raman spectroscopy: a powerful stand-off spatial offset Raman spectroscopy for the detection of concealed content in distant objects," Analytical Chemistry, vol. 83, no. 24, pp. 9438-9442, 2011.

[18] E. D. Emmons, A. Tripathi, J. A. Guicheteau, S. D. Christesen, and A. W. Fountain, "Raman chemical imaging of explosivecontaminated fingerprints," Applied spectroscopy, vol. 63, no. 11, pp. 1197-1203, 2009.

[19] B. Schrader, A. Hoffmann, A. Simon, and J. Sawatzki, "Can a Raman renaissance be expected via the near-infrared Fourier transform technique?" Vibrational Spectroscopy, vol. 1, no. 3, pp. 239-250, 1991.

[20] D. B. Chase, "Fourier transform Raman spectroscopy," Journal of the American Chemical Society, vol. 108, no. 24, pp. 74857488, 1986.

[21] D. A. Long, Raman Spectroscopy, McGraw-Hill, New York, NY, USA, 1977.

[22] S. D. Williams, T. J. Johnson, T. P. Gibbons, and C. L. Kitchens, "Relative Raman intensities in $\mathrm{C}_{6} \mathrm{H}_{6}, \mathrm{C}_{6} \mathrm{D}_{6}$, and $\mathrm{C}_{6} \mathrm{~F}_{6}$ : a comparison of different computational methods," Theoretical Chemistry Accounts, vol. 117, no. 2, pp. 283-290, 2007.

[23] T. A. Blake, J. F. Kelly, N. B. Gallagher, P. L. Gassman, and T. J. Johnson, "Passive standoff detection of RDX residues on metal surfaces via infrared hyperspectral imaging," Analytical and Bioanalytical Chemistry, vol. 395, no. 2, pp. 337-348, 2009.

[24] T. Okuno, "Thermal effect of visible light and infra-red radiation (IR-A, IR-B AND IR-C) on the eye-a study of infra-red cataract based on a model," Annals of Occupational Hygiene, vol. 38, no. 4, pp. 351-359, 1994.

[25] S. J. Choquette, E. S. Etz, W. S. Hurst, D. H. Blackburn, and S. D. Leigh, "Relative intensity correction of Raman spectrometers: NIST SRMs 2241 through 2243 for $785 \mathrm{~nm}, 532 \mathrm{~nm}$, and $488 \mathrm{~nm} / 514.5 \mathrm{~nm}$ excitation," Applied Spectroscopy, vol. 61, no. 2, pp. 117-129, 2007.

[26] M. Fryling, C. J. Frank, and R. L. McCreery, "Intensity calibration and sensitivity comparisons for CCD/Raman spectrometers," Applied Spectroscopy, vol. 47, pp. 1965-1974, 1993.

[27] K. G. Ray and R. L. McCreery, "Simplified calibration of instrument response function for Raman spectrometers based on luminescent intensity standards," Applied Spectroscopy, vol. 51, no. 1, pp. 108-116, 1997.

[28] R. Martini, C. Glazowski, E. A. Whittaker et al., "Optical freespace communications at middle infra-red wavelengths," in Quantum Sensing and Nanophotonic Devices, M. Razeghi and G. J. Brown, Eds., vol. 5359 of Proceedings of SPIE, pp. 196202, San Jose, Calif, USA, January 2004.

[29] A. W. Fountain III, C. K. Mann, and T. J. Vickers, "Routine wavenumber calibration of an FT-Raman spectrometer," Applied Spectroscopy, vol. 49, no. 7, pp. 1048-1053, 1995.

[30] W. S. Hurst, S. J. Choquette, and E. S. Etz, "Requirements for relative intensity correction of Raman spectra obtained by column-summing charge-coupled device data," Applied Spectroscopy, vol. 61, no. 7, pp. 694-700, 2007.

[31] P. Vandenabeele, "Evaluation of a spectral searching algorithm for the comparison of Raman band positions," Spectrochimica Acta A, vol. 80, no. 1, pp. 27-31, 2011.

[32] R. J. Bell, Introductory Fourier Transform Spectroscopy, Academic Press, New York, NY, USA, 1972.

[33] S. W. Sharpe, R. L. Sams, T. J. Johnson, P. M. Chu, G. C. Rhoderick, and F. R. Guenther, "Creation of $0.10 \mathrm{~cm}^{-1}$ resolution, quantitative, infrared spectral libraries for gas samples," in Vibrational Spectroscopy-based Sensor Systems, vol. 4577 of Proceedings of SPIE, pp. 12-24, Boston, Mass, USA, 2002. 
[34] S. W. Sharpe, T. J. Johnson, R. L. Sams, P. M. Chu, G. C. Rhoderick, and P. A. Johnson, "Gas-phase databases for quantitative infrared spectroscopy," Applied Spectroscopy, vol. 58, no. 12, pp. 1452-1461, 2004.

[35] J. E. Bertie, "Specification of components, methods, and parameters in Fourier transform spectroscopy by Michelson and related interferometers: IUPAC recommendations," Pure and Applied Chemistry, vol. 70, no. 10, pp. 2039-2045, 1998.

[36] B. Schrader, A. Hoffmann, A. Simon, R. Podschadlowski, and M. Tischer, "NIR-FT-Raman-spectroscopy, state of the art," Journal of Molecular Structure, vol. 217, pp. 207-220, 1990.

[37] T. J. Johnson, R. L. Sams, T. A. Blake, S. W. Sharpe, and P. M. Chu, "Removing aperture-induced artifacts from Fourier transform infrared intensity values," Applied Optics, vol. 41, no. 15, pp. 2831-2839, 2002.

[38] D. Bonen, T. J. Johnson, and S. L. Sarkar, "Characterization of principal clinker minerals by FT-Raman microspectroscopy," Cement and Concrete Research, vol. 24, no. 5, pp. 959-965, 1994.

[39] J. W. C. Johns, "High resolution and the accurate measurement of intensities," Mikrochimica Acta, vol. 93, no. 1-6, pp. 171-188, 1987.

[40] A. G. Maki and J. S. Wells, "Wavenumber calibration tables from heterodyne frequency measurements," National Institute of Standards and Technology, Special Publication 821, U.S. Government Printing Office, Washington, DC, 1991, http://physics.nist.gov/PhysRefData.

[41] D. Hutsebaut, P. Vandenabeele, and L. Moens, "Evaluation of an accurate calibration and spectral standardization procedure for Raman spectroscopy," Analyst, vol. 130, no. 8, pp. 12041214, 2005.

[42] A. W. Fountain III, T. J. Vickers, and C. K. Mann, "Factors that affect the accuracy of Raman shift measurements on multichannel spectrometers," Applied Spectroscopy, vol. 52, no. 3, pp. 462-468, 1998.

[43] M. Fryling and C. J. Frank, "Intensity calibration and sensitivity comparisons for CCD/Raman spectrometers," Applied Spectroscopy, vol. 47, no. 12, pp. 1965-1974, 1993.

[44] "Standard Guide for Testing the Resolution of a Raman Spectrometer," ASTM E2529-06, ASTM Publication, www.astm .org/.

[45] J. B. Forrester, N. B. Valentine, Y.-F. Su, and T. J. Johnson, "Chemometric analysis of multiple species of Bacillus bacterial endospores using infrared spectroscopy: discrimination to the strain level," Analytica Chimica Acta, vol. 651, no. 1, pp. 24-30, 2009.

[46] B. M. Kunkel, Y.-F Su, and R. G. Tonkyn, "Raman database considerations for near-infrared systems," in Optics and Photonics for Counterterrorism and Crime Fighting VII, vol. 8189, article 818905 of Proceedings of SPIE, Prague, Czech Republic, September 2011.

[47] I. Geiman, M. Leona, and J. R. Lombardi, "Application of Raman spectroscopy and surface-enhanced Raman scattering to the analysis of synthetic dyes found in ballpoint pen inks," Journal of Forensic Sciences, vol. 54, no. 4, pp. 947-952, 2009.

[48] M. L. Lewis, I. R. Lewis, and P. R. Griffiths, "Anti-Stokes Raman spectrometry with 1064-nm excitation: an effective instrumental approach for field detection of explosives," Applied Spectroscopy, vol. 58, no. 4, pp. 420-427, 2004.

[49] N. W. Daniel Jr., I. R. Lewis, and P. R. Griffiths, "Interpretation of Raman spectra of nitro-containing explosive materials. Part II: the implementation of neural, fuzzy, and statistical models for unsupervised pattern recognition," Applied Spectroscopy, vol. 51, no. 12, pp. 1868-1879, 1997.

[50] D. Patel, C. Vance, N. King, M. Jessup, and S. Sarkisov, "Preparation, fluorescence spectroscopy and AFM analysis of erbium oxide nanocolloid," in Optical Components and Materials VI, vol. 7212, article 72121F of Proceedings of SPIE, 2009.

[51] S. Heer, K. Kömpe, H.-U. Güdel, and M. Haase, "Highly efficient multicolour upconversion emission in transparent colloids of lanthanide-doped $\mathrm{NaYF}_{4}$ nanocrystals," Advanced Materials, vol. 16, no. 23-24, pp. 2102-2105, 2004. 


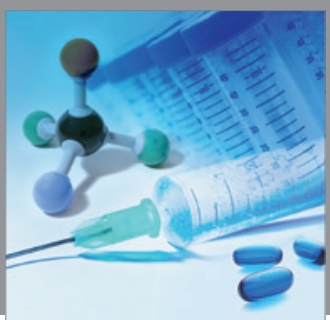

International Journal of

Medicinal Chemistry

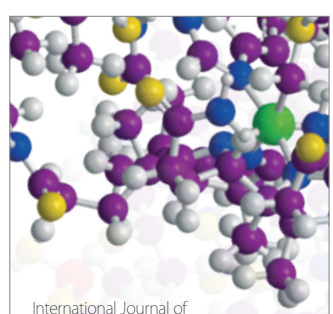

Carbohydrate Chemistry

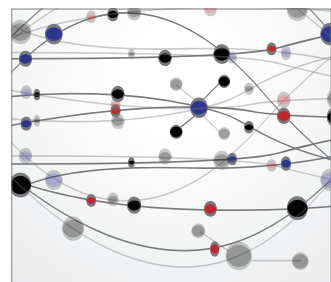

The Scientific World Journal
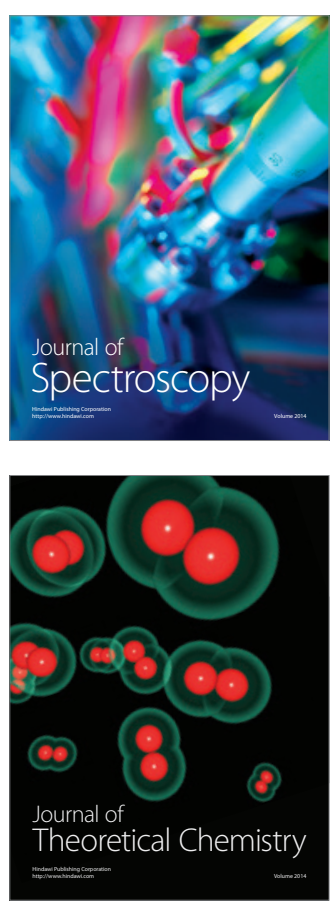
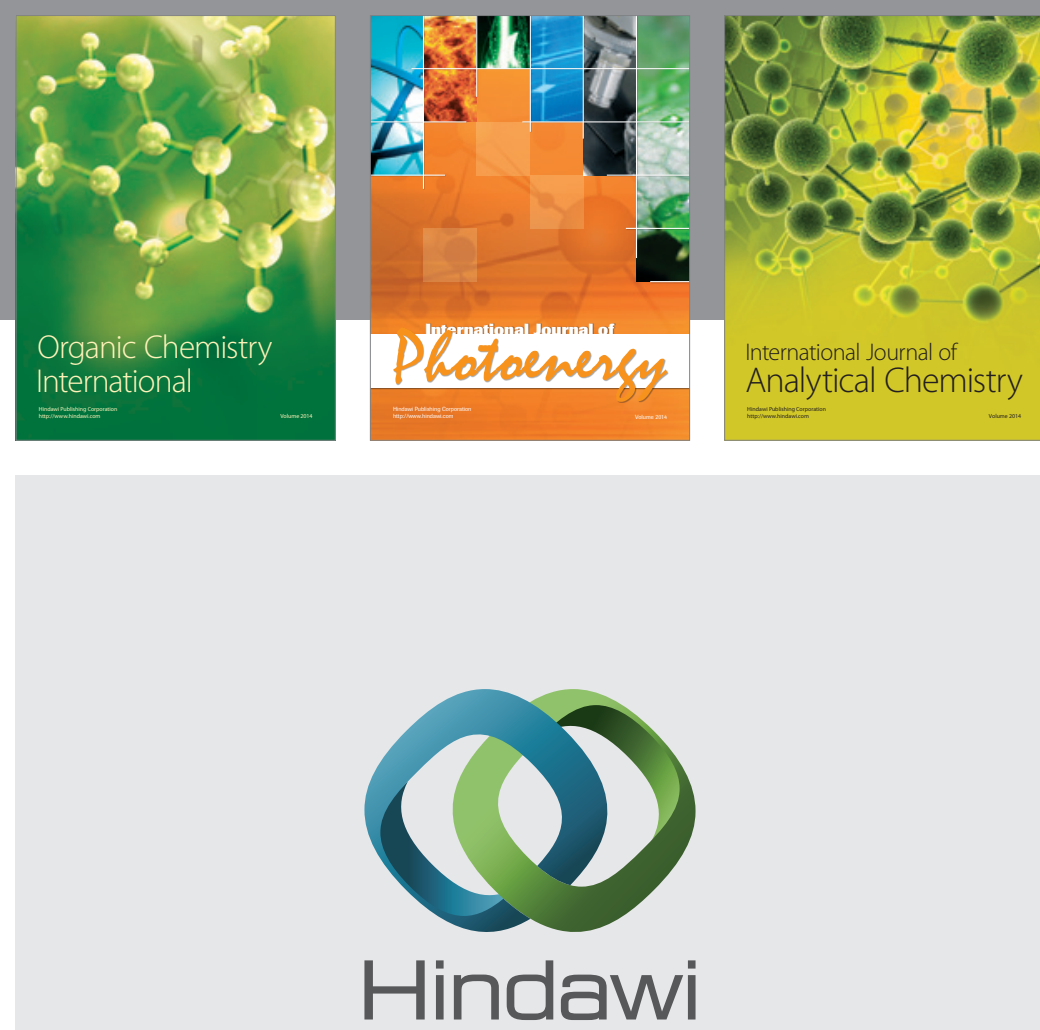

Submit your manuscripts at

http://www.hindawi.com
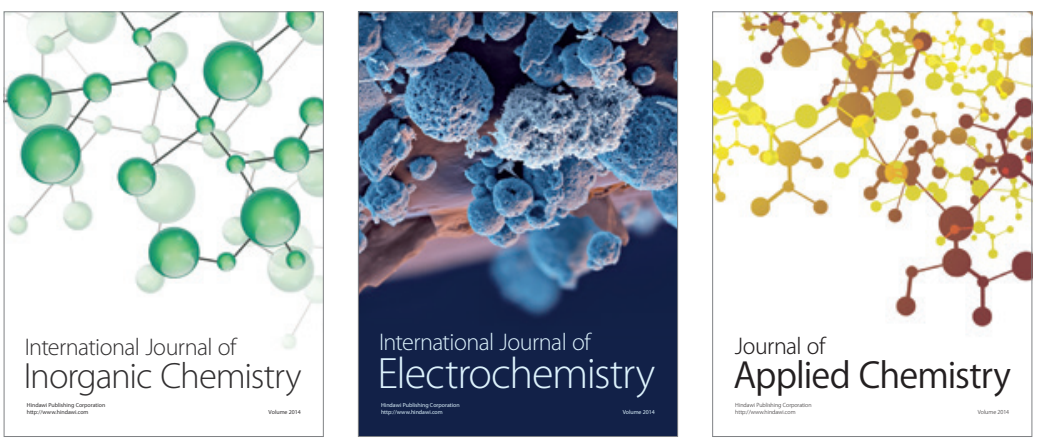

Journal of

Applied Chemistry
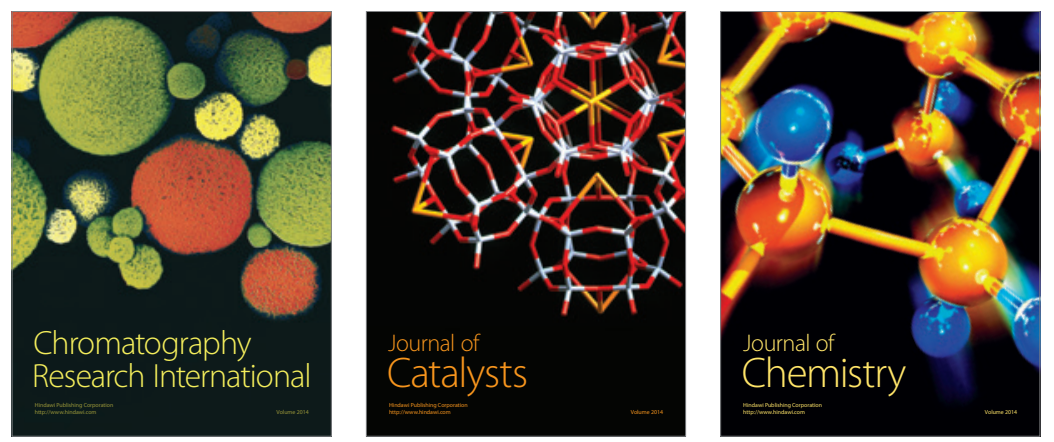
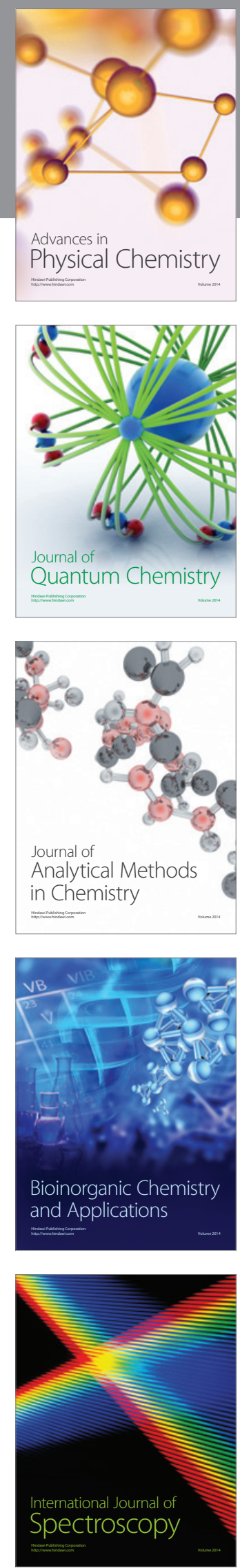